\title{
An information processing view of framing effects: The role of causal schemas in decision making
}

\author{
JERWEN JOU \\ University of Texas-Pan American, Edinburg, Texas \\ and \\ JAMES SHANTEAU and RICHARD JACKSON HARRIS \\ Kansas State University, Manhattan, Kansas
}

\begin{abstract}
People prefer a sure gain to a probable larger gain when the two choices are presented from a gain perspective, but a probable larger loss to a sure loss when the objectively identical choices are presented from a loss perspective. Such reversals of preference due to the context of the problem are known as framing effects. In the present study, schema activation and subjects' interpretations of the problems were examined as sources of the framing effects. Results showed that such effects could be eliminated by introducing into a problem a causal schema that provided a rationale for the reciprocal relationship between the gains and the losses. Moreover, when subjects were freed from framing they were consistently risk seeking in decisions about human life, but risk averse in decisions about property. Irrationality in choice behaviors and the ecological implication of framing effects are discussed.
\end{abstract}

The same information presented in different forms can lead to different decisions. Changes in decision associated with different presentation forms are known as framing effects. For example, people's preference of choices can reverse as a function of the form in which logically identical questions are represented (Bradburn, 1982; Goldstein \& Einhorn, 1987; Hershey \& Schoemaker, 1980; Levin, Schnittjer, \& Thee, 1988; Lichtenstein \& Slovic, 1973; Redelmeier \& Tversky, 1992; Schneider, 1992; Schneider \& Lopes, 1986; Slovic, Fischhoff, \& Lichtenstein, 1982; Tversky \& Kahneman, 1981). Tversky and Kahneman (1981; Kahneman \& Tversky, 1979) demonstrated that people showed a risk aversion or a risk-seeking preference in choice behavior under uncertain circumstances, depending on whether a problem was presented in a gaining (positive) or a losing (negative) frame. A frame provides a reference point from which a choice problem is represented to the subjects (Dawes, 1988; Tversky \& Kahneman, 1981). For instance, when given a choice between a sure gain of some-

Several precursors of this study were presented at the 1991 and 1992 Psychonomic Society meetings, and at the 1993 American Psychological Society meeting. We thank all the anonymous reviewers for helpful critiques, comments, and suggestions which led to substantial improvement of this paper. We are especially grateful to Tony Greenwald for his constructive critiques and helpful suggestions on an earlier version of the paper. Special thanks are due Douglas Hintzman for his helpful comments and detailed editing. Thanks are also due Robert Proctor for useful comments, and Christina Salinas at UTPA for her help with the experiments. Correspondence concerning this paper should be addressed to J. Jou, Department of Psychology and Anthropology, University of TexasPan American, Edinburg, TX 78539-2999 (e-mail: jj809b@) anam1. panam.edu) thing and an uncertain but possibly larger gain (with equal or larger expected value), the majority of people prefer the sure gain alternative to the uncertain gamble - the tendency called risk aversion. However, when the logically equivalent choices are presented from a losing perspective, the majority of people tend to choose the risky gamble over the sure-loss alternative-the tendency called risk seeking (Tversky \& Kahneman, 1981).

Kahneman and Tversky (1979) proposed prospect theory to explain the bias in these choice behaviors. According to this theory, people value a certain gain more than a probable gain with equal or greater expected value; the opposite is true for losses. Moreover, gains and losses are evaluated from a subjective reference point. However, the function relating the subjective value and the corresponding losses is steeper than that for the gains. As a result, the displeasure associated with the loss is greater than the pleasure associated with the same amount of gains. Therefore, people respond differently, depending on whether the choices are framed in terms of gains or losses.

Such a dependence on a frame of reference has been compared to an analogous phenomenon in perceptual judgment, the dependence of a percept on perspective (Einhorn \& Hogarth, 1981; Helson, 1948; Tversky \& Kahneman, 1981). The framing effect in a broader sense can be demonstrated under a variety of contexts. A widely cited typical example of the framing effect in choice making is Tversky and Kahneman's (1981) disease problem. In the present study, we used this example as a framework in order to explore some possible cognitive causes of the framing effect and to suggest an alternative perspective for looking at this phenomenon. 
The positive form of the disease problem states that the United States is preparing for the outbreak of an unusual disease, which is expected to kill 600 people. Two alternative programs to combat the disease have been proposed. Program A will save 200 lives, and Program B will provide a $1 / 3$ probability of saving 600 people and a $2 / 3$ probability of saving no people. The negative form of the problem presented the two choices from a losing point of view: Program $C$ will result in the death of 400 people, whereas Program D offers a $1 / 3$ probability that nobody will die, and a $2 / 3$ probability that 600 people will die (for the disease problem and other problems used in this study, see the Appendix). In the positive frame, the majority of subjects ( $72 \%$ ) chose Alternative A over Alternative B, demonstrating risk aversion. In the negative frame, the majority of subjects $(78 \%)$ chose Alternative $\mathrm{D}$, demonstrating risk seeking.

According to Tversky and Kahneman (1981), on the basis of what was stated in the cover story of the problem, saving 200 lives implies losing 400 lives, and similarly, losing 400 lives implies saving 200 lives. Thus, Programs $\mathrm{A}$ and $\mathrm{C}$ were logically equivalent, as were Programs B and $\mathrm{D}$. Yet the preference was reversed across the two frames. Therefore, the subjects' choices, according to Tversky and Kahneman, demonstrated susceptibility of their judgments to problem framing. The effect was so robust that even when a subject was asked to do both versions of the problem (in a within-subjects design), the effect was not eliminated (Dawes, 1988; Kahneman \& Tversky, 1986). Hence, framing is more than simple verbal trickery (Dawes, 1988). The framing effect was taken as an indication of a violation of the logical consistency or invariance principle of rational decision making (Kahneman \& Tversky, 1986; Tversky \& Kahneman, 1981). According to the invariance principle, the choices should not reverse simply because of the way in which the questions are phrased. Yet the framing effects showed that people are logically inconsistent in their choice behavior.

Kahneman and Tversky's (1979) prospect theory gave a mathematical account for risk aversion in the gain frame and risk seeking in the loss frame. It did not, however, address the question of why people were controlled by framing in their judgments, other than the passing mention that the bias derived from norms, habits, and personal characteristics of the decision maker (Kahneman \& Tversky, 1979; Tversky \& Kahneman, 1981). Prospect theory did not give an explanation for why people cannot see the other side of a decision consequence when they are presented with one frame. This question becomes even more interesting in view of the persistence of the effects in a withinsubjects design. The purpose of this study is not to dispute the fact that people showed reversed tendencies under two frames. Rather, the goal is to explain why they are limited to only one perspective under the circumstances of Tversky and Kahneman's original study, and to question the view that these tendencies are prevalent and irrational.

The central question asked in the present study is this: Is Tversky and Kahneman's (1981) result a demonstration of fundamental inconsistency in human judgment, or is it simply a manifestation of a disagreement between the experimenters' and the subjects' interpretations of the problem (Berkeley \& Humphreys, 1982; Cohen, 1981; Hogarth, 1981; Jungermann, 1986)? The crux of the issue is the assumption that saving 200 lives is equivalent to losing 400 lives, and that losing 400 lives is equivalent to saving 200 lives. According to the way in which the problem was phrased in Tversky and Kahneman's original study, is there a legitimate basis for positing such a mutually exhaustive and exclusive relationship between lives saved and lives lost? In other words, is there enough information given in the original problem to establish a common understanding of a relationship as laid out in the following formula?

$$
\begin{aligned}
& \text { Total expected loss }- \text { Saved }=\text { Resulting loss } \\
& \text { Total expected loss }- \text { Actual loss }=\text { Saved }
\end{aligned}
$$

We argue that the equivalence relationship as defined in Tversky and Kahneman's (1981) original problem is arbitrary in the sense that it does not give a rationale for such a relationship between the gain and the loss which corresponds to people's familiar life experience.

Cheng and Holyoak (1985) found that subjects' performance on selection problems (involving the use of the logical rule "if ... then ...") could be substantially improved when the problem was embedded in a familiar schema that provided a rationale for the rule. For instance, when subjects were asked to turn over a minimal number of four cards labeled "E," "K," "4," and "7," respectively, to verify the conditional rule "If a card has a vowel on one side, then it has an even number on the other side," their performance was poor (Wason, 1966). But when the same rule was tested in the context of a "prerequisite" schema (e.g., "if someone wants to enter a country, he or she should have had some requirements met-e.g., inoculations"), the performance substantially improved (Cheng $\&$ Holyoak, 1985). Similarly, when the same problem was embodied in a familiar everyday event (e.g., "If one drinks beer, then one is over 19" [U.S.A.]; or "If a letter is sealed, then it has a 50-lire stamp on it" [Italy]), the performance also improved significantly (Cox \& Griggs, 1982; JohnsonLaird, Legrenzi, \& Legrenzi, 1972; Wason \& JohnsonLaird, 1972).

People have prototypical knowledge about certain types of events and comprehend the relationship between events by referring to such general knowledge structures known as schemata. When encountered events cannot be fit into a schema, the relationship between the events will not be understood (Graesser \& Nakamura, 1982; Shank \& Abelson, 1977). Given the original form of the disease problem presented by Tversky and Kahneman (1981), people may not have understood the gain-loss equivalence relationship defined in Equations 1 and 2. Since no circumstances were described in the original problem that would match the schema of such an equivalence relationship, people may have constructed a mental representation regarding the consequence of the choice differently than the experimenters had intended (Berkeley \& Humphreys, 1982; Einhorn \& Hogarth, 1981; Jungermann, 1986). 
Such a different representation in turn might lead to different decisions. For example, in the context of the disease problem, the deaths could be construed as occurring immediately after the decision to save 200 lives, or at some indefinite future time. If the deaths were construed as occurring at some unknown future time, they would not likely be seen as a consequence of saving 200 lives. Hence, saving the lives will not be conceived as entailing the death of 400 people. Similarly, subjects may not be able to imagine how causing the death of 400 people (in the negative frame) could mean saving 200 people. The assumed mutual causal relationship between the gain and the loss may not have been conveyed in Tversky and Kahneman's (1981) original disease problem in a medium that could invoke any of the subjects' schemata or scripts (Schank \& Abelson, 1977) about such a relationship. Instead, it was implicitly assumed by the experimenters. Yet the conclusion of inconsistency or irrationality was based on this premise. To use Tversky and Kahneman's (1973) concept of availability, we suggest that the way in which the original problem was presented created low availability of the reciprocal consequence in the subjects' mental representation of the problem, owing to its failure to activate a relevant schema. By raising the availability level of the other consequence through invoking a familiar schema, the framing effects may be largely reduced or even eliminated.

In this study, we introduced a description of a situation into each scenario, the underlying theme of which suggested a limitation condition-that because of a limitation in time, space, resources, opportunities, or otherwise, saving some proportions of lives or property would necessitate sacrificing other lives or property. Alternatively, if one did not want to take this option, one could take the risky option of saving or losing all. For example, one might either give the only full dose of a life-saving medicine to Patient A and save her life, but let Person B die, or divide the limited dose in half at a risk of insufficient dosage for both. This limitation theme can be termed a causal schema, since it links choice to outcome and interrelates positive and negative consequences. People encounter such circumstances often in everyday life.

Our hypothesis was that the causal schema would (1) give a rationale (explanatory mechanism) for why and how saving some lives is equal to losing other lives (equivalence relationship), and as a result, would (2) mentally activate the other side of the consequence of the decision, which would lead to (3) constraining the interpretation of the problem (i.e., making subjects' representation of the problem better match the experimenter's). On the basis of this hypothesis, we predicted that subjects' responses in the positive and negative frames would no longer be inconsistent when the limitation theme was built into the scenario.

We also predicted that the reciprocal consequence would be used in the decision-making processes more frequently when the causal schema was present than in the original form of the problem. Introducing the causal schema should increase the cognitive accessibility of the other side of the consequence.

\section{EXPERIMENT 1}

Four endangered-life and four endangered-property problems, each presented in two forms, were used in Experiment 1 . The first was modeled after Tversky and Kahneman's (1981) original disease problem, which is referred to here as the no-rationale condition. In the second form, the rationale condition, a causal schema explained the equivalence relationship of the two reciprocal consequences. The limitation situations used were all familiar, easily understandable events that corresponded to people's everyday life experiences.

In each condition, subjects were asked to give the reason(s) for their choices. This information was collected in order to determine how frequently subjects take into consideration both sides of the consequence as the basis for their choices.

\section{Method}

Subjects. A total of 320 students participated for extra credit in introductory psychology courses at the University of Texas-Pan American. Eighty of them served in each of the four conditions: norationale positive frame, no-rationale negative frame, rationale positive frame, and rationale negative frame.

Materials and Design. The four endangered-life problems involved (1) an adaptation of Tversky and Kahneman's (1981) original disease scenario, (2) a shipwreck, (3) a volcanic explosion, and (4) a hurricane scenario. The four endangered-property problems involved (1) a shipwreck (with precious metal endangered), (2) investment, (3) renting an apartment, and (4) selling business assets. The no-rationale condition gave only the total amount of life or property in danger. The rationale condition described, in addition, situational limitations that explained why it was impossible to save all life or property and why some could be saved only at the expense of others. For example, in the disease problem, it was stated that 600 people had contracted a fatal disease and were sure to die without treatment. However, there was a limited supply of the medicine, enough to save only 200 patients' lives. If the medicine were divided among all 600 patients, there would be a $1 / 3$ probability that all 600 patients would be saved and a $2 / 3$ probability that none would be saved, because of insufficient dosage. The probabilities used were uniformly $1 / 3$ and $2 / 3$. The total amounts of life or property in danger was given as 600 if this number was not unreasonable in the given context, or as another more plausible number. Only one problem in the endangered-life condition (a hurricane with fewer than 600 lives in danger), and two problems in the endangered-property condition (investment and selling assets, each with property value of more than $\$ 600$ at risk) had a value other than 600 .

Four independent variables were manipulated: rationale (no rationale vs. rationale, between subjects), entity (life vs. property, within subjects), scenario (four different life-in-danger scenarios nested under the life condition and different property-in-danger scenarios nested under the property condition, within subjects), and frame (positive vs. negative, between subjects). The whole set of the problems is presented in the Appendix

Procedure. The subjects were administered the eight problems (four life in danger, and four property in danger) in group sessions. Each problem was printed on a separate sheet, and the nine sheets (the first being an instruction page) were stapled together, with the eight problems in a different random order for each subject. The numbers of subjects assigned to the between-subjects conditions at each session were made as equal as possible.

The following instructions were given to the subjects:

This experiment involves asking you to make some decisions in some hypothetical situations with lives and properties in danger. You should 
treat each one of the problems as an independent problem, which means that you should judge each problem without being affected by the decisions you have already made for the previous problems. Once a certain page has been turned, you may not return to it or any other earlier pages to make any change. Your estimate of the likelihood of the occurrence of some event should be strictly based on the probabilities given you in each problem, not based on your personal intuitions or experience about the likelihood of a certain event's occurring. Also, when you make these decisions, assume that you are not personally involved in the situations, and that you will be kept anonymous. Please make what you think are the best decisions. And for each choice you make, state the reason or reasons why you make that choice. There is no time limit for completing this questionnaire. Finally, before you give the answers, please be sure to read the cover story and the choices very carefully. Are there any questions?

The experimenter read the instructions aloud while the subjects were reading them.

\section{Results}

Two dependent measures were analyzed, the percentage of risk-averse responses, and the percentage of reciprocal answers to the last question in the questionnaire about the reason for choosing or not choosing a certain alternative. A reciprocal answer was defined as one in which the reciprocal consequence was cited as the reason for choosing or not choosing an alternative. The purpose in analyzing the first measure was to test the hypothesis that the rationale introduced would substantially reduce or eliminate the framing effect. The aim in analyzing the percent of reciprocal answers was to test the hypothesis that the proportion of the reciprocal answers given would be significantly higher in the rationale condition than in the norationale condition.

Analysis of the percentage of risk-averse choice. The percentages of the risk-averse choices for each problem under each frame in the no-rationale condition and the rationale condition are presented in Figures 1 and 2, respectively. A small number of subjects declined to make a choice for certain questions because they preferred none

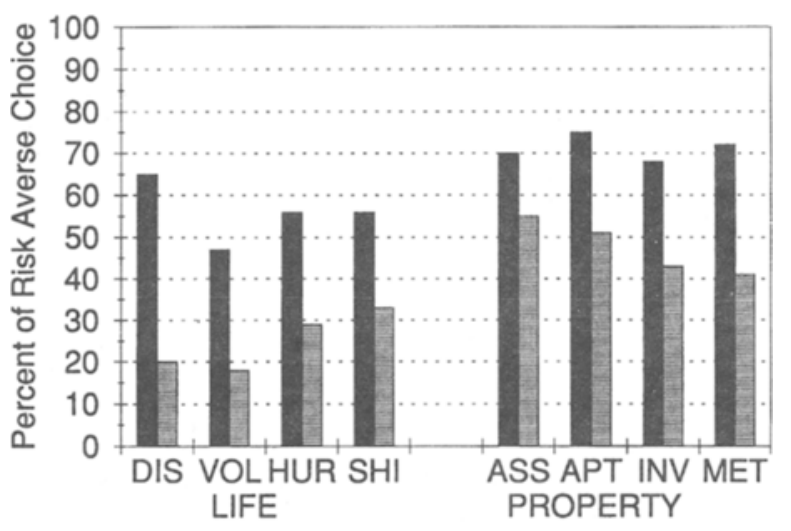

\section{POSITIVE FRAME NEGATIVE FRAME}

Figure 1. Percentages of risk-averse choice for each scenario in each frame in the no-rationale condition of Experiment 1. Dis, disease; Vol, volcano; Hur, hurricane; Shi, shipwreck, life; Ass, selling assets; Apt, apartment; Inv, investment; Met, shipwreck, metal.

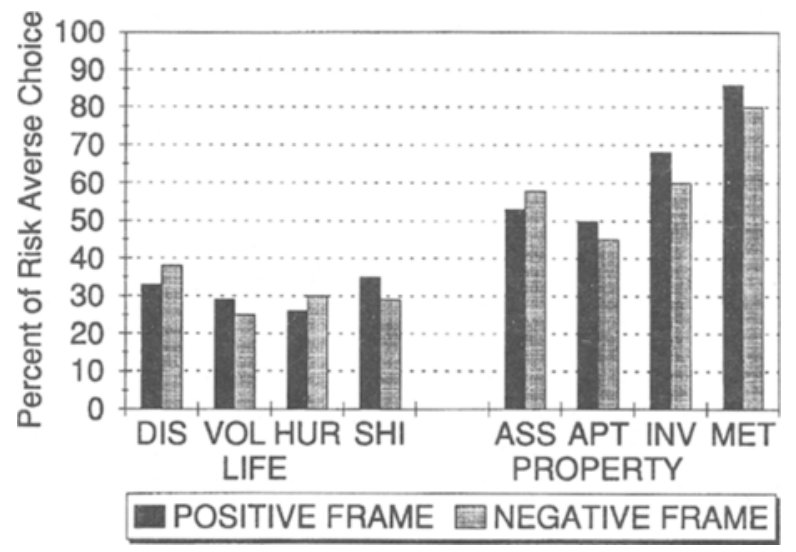

Figure 2. Percentages of risk-averse choice for each scenario in each frame in the rationale condition of Experiment 1. Dis, disease; Vol, volcano; Hur, hurricane; Shi, shipwreck, life; Ass, selling assets; Apt, apartment; Inv, investment; Met, shipwreck, metal.

of the options. Consequently, the $N$ on which a mean was based could range from 78 to 80 . An analysis of variance for categorical data was performed (Categorical Data Modeling or CATMOD in SAS, using a log-linear analysis. For the method of analysis, see SAS Institute, 1985). Only significant $(p<.05)$ effects are reported. The entity main effect was significant $\left[\chi^{2}(1)=166.24, p<.001\right]$, with the mean for the life condition being 36 , and for the property condition, 61 . Thus, overall, people are more risk seeking in dealing with life than with properties.

The scenario main effect was also significant (see Figures 1 and 2 for the scenario means) $\left[\chi^{2}(6)=19.01, p<\right.$ $.001]$. The frame main effect was significant $\left[\chi^{2}(1)=\right.$ $55.57, p<.001]$, with the mean for the positive frame being 58 , and for the negative, 41 . Thus, the positive frame produced a greater proportion of risk-averse responses than did the negative frame, which is consistent with the traditional framing effects.

The effect crucial to the hypothesis is the rationale $x$ frame interaction, which was significant $\left[\chi^{2}(1)=41.47\right.$, $p<.001]$. In the no-rationale condition, the mean percent of risk-averse choice was 64 for the positive frame, and 36 for the negative frame. In the rationale condition, these two percentages were 48 and 46 . A separate analysis was performed to determine whether the frame effect was significant in the no-rationale condition but not in the rationale condition. For the no-rationale condition, the framing effect was significant $\left[\chi^{2}(1)=96.86, p<.001\right]$, whereas for the rationale condition, it was not $\left[\chi^{2}(1)=.51, p>\right.$ $.05]$. Thus, the introduction of the causal schema eliminated the framing effects.

This is an exemplary case of a statistical interaction indicating that a theory (the prospect theory in this case) fails to hold under a different situation (Greenwald, Pratkanis, Leippe, \& Baumgardner, 1986). The rationale $X$ entity interaction was significant $\left[\chi^{2}(1)=9.71, p<.005\right]$. This interaction derived from the fact that the difference in response between life in danger and property in danger 
increased from the no-rationale to the rationale condition. In other words, with the rationale introduced, the responses to life problems became consistently more risk seeking, whereas those to the property problems became more risk averse. The rationale $\times$ scenario interaction was significant $\left[\chi^{2}(6)=12.83, p<.05\right]$. No other effect was significant.

Analysis of the percentage of reciprocal answers. If the subject's answer to the question of why a certain alternative was chosen explicity cited the reciprocal consequence as the reason for choosing or not choosing the alternative, it was classified as a reciprocal answer. For example, in a positive frame, if a subject did not choose Alternative A (e.g., save 200 lives), he or she might ask "Who is to decide which 400 people should die?" or say "I wouldn't be able to live with myself knowing that I let 400 people die," or "I would rather take a chance to save all of them than sending 400 of them to death," and so forth. In the negative frame, if they chose Alternative A (e.g., lose 400 lives), they might say "Better to assure the lives of 200 people than risk the probable death of so many," "This insures at least 200 people will survive," "For sure that 200 boxes won't be lost," and so on. If a subject did not choose Alternative A, he or she might say "Because she stands to lose more than she stands to gain. She will only get back $\$ 200$ which isn't much today, so she should try for the $\$ 600$ total cash back..."

The main effect of rationale was significant $\left[\chi^{2}(1)=\right.$ $109.53, p<.001]$. The mean percent of reciprocal answers was 19 for the no-rationale condition, and 38 for the rationale condition; this confirmed our hypothesis that subjects would use the reciprocal side of the information to a greater extent in the rationale condition than in the norationale condition. The main effect of scenario was significant $\left[\chi^{2}(6)=14.56, p<.001\right]$. These mean percentages are presented in Table 1 . The main effect of frame was significant $\left[\chi^{2}(1)=5.73, p<.05\right]$. The mean percent of reciprocal answers was 27 for the positive frame, and 31 for the negative frame; this indicates that overall, subjects gave a slightly but significantly higher percent of reciprocal answers in the negative than in the positive frame.

The entity $\times$ frame interaction was significant $\left[\chi^{2}(1)=\right.$ $27.06, p<.001]$. The interaction reflects the fact that the reciprocal answers were more frequent under the positive frame than under the negative frame for the life-in-danger scenarios ( 30 vs. 24 ); the relative magnitudes were reversed for the property-in-danger scenarios ( 24 vs. 37$)$.

\section{Discussion}

Both hypotheses were supported by the results. First, the introduction of a causal schema which served as a rationale for the equivalence relationship between gains and losses eliminated the framing effects. Second, such a rationale increased the probability that the other side of the consequence would be evoked or used as a basis for the choice. These results support the theory that subjects in the no-rationale condition may not have represented the consequence of the decision in the form of the equivalence relationship as defined in Equations 1 and 2. When they represented the problems in light of the equivalence relation (as shown by their more frequent access of the reciprocal consequence in their decision process), the framing effects disappeared. The low frequency of mentioning the reciprocal consequences in the no-rationale condition suggests that the equivalence relationship was, in most cases, not represented or activated. However, when the relationship could be fit into a schema and therefore mentally represented, subjects no longer showed the inconsistency.

From the point of view of information processing, the way in which a problem was presented in the no-rationale condition highlighted some information (positive or negative) and obscured the other. What was done in our manipulation was to more clearly define the other aspect through the use of a schematic theme. As a result, subjects were more likely to take both sides of the consequence of a decision into consideration in their decision process regardless of the frame. Instead of being dominated by one aspect of the information, subjects in the rationale condition may have formed an integrated memory representation of the whole event.

A systematic pattern in the data common across the two rationale conditions was that overall, choices concerning human life were more risk seeking than choices concerning property. This pattern may be a reflection of a general reluctance on the part of the subjects to decide "who shall live and who shall die." It may be considered unfair to save some lives at the expense of others. In fact, a common reason given by subjects for not choosing the certain alternative in the life-in-danger scenarios was that all people should have an equal chance to be saved. For the property, it seemed acceptable to sacrifice some in order to salvage

Table 1

Percentages of Reciprocal Answers Given Spontaneously by Subjects in Experiment 1

\begin{tabular}{|c|c|c|c|c|c|c|c|c|c|}
\hline \multirow[b]{2}{*}{ Condition } & \multicolumn{4}{|c|}{ Life } & \multicolumn{4}{|c|}{ Property } & \multirow[b]{2}{*}{$M$} \\
\hline & Dis & Vol & Hur & Shi & Ass & $\mathrm{Apt}$ & $\operatorname{Inv}$ & Met & \\
\hline \multicolumn{10}{|l|}{ No-rationale } \\
\hline Positive & 13 & 28 & 14 & 25 & 10 & 8 & 19 & 18 & 16.9 \\
\hline Negative & 15 & 16 & 15 & 22 & 24 & 27 & 26 & 30 & 21.9 \\
\hline \multicolumn{10}{|l|}{ Rationale } \\
\hline Positive & 43 & 35 & 34 & 43 & 39 & 31 & 35 & 34 & 36.8 \\
\hline Negative & 36 & 23 & 32 & 35 & 44 & 37 & 44 & 66 & 39.6 \\
\hline
\end{tabular}

Note-Dis, disease; Vol, volcano; Hur, hurricane; Shi, shipwreck, life; Ass, selling assets; Apt. apartment; Inv, investment; Met, shipwreck, metal. 
others; as many subjects said in their answers, "Money is money." Thus, when people are given a forced choice between a conservative and a risky alternative, they seem to use different principles regarding human lives and properties. In the no-rationale condition, there was a weak but discernible pattern of more risk seeking toward human lives than toward properties. This tendency became more pronounced and systematic in the rationale condition. All the means for the life scenarios fell below the $50 \%$ line, but all except one mean (the apartment problem) of the property scenarios fell above the $50 \%$ line. For the apartment problem, subjects seemed to show no clear preference for either choice.

The differential response tendency in dealing with life and property problems might be explained by the evocation of the pragmatic schema introduced into the scenarios. The subjects followed certain behavioral norms in stereotypical situations. Such norms specify that human lives are not interchangeable, but that properties are. In the norationale condition, the equivalence relationship was not recognized by the subjects; therefore, the issue of interchangeability did not occur, and the different norms were hence irrelevant.

Another aspect of the data is worth mentioning. Although the framing effects in the no-rationale condition were evident, they were not always in the form observed in Tversky and Kahneman's (1981) original study - that is, risk averse in the positive frame (over $50 \%$ of risk-averse responses), and risk seeking in the negative frame (less than $50 \%$ of risk-averse responses). This deviation from Tversky and Kahneman's pattern was especially pronounced for the property problems. For some problems, both the positive and negative frames showed a risk-averse tendency (over $50 \%$ risk-averse choices), only one more so than the other. This discrepancy from the traditional strong form of framing effects might be attributable to different scenarios and experimental procedures in our study; entity and scenarios were both within-subjects variables, whereas in Tversky and Kahneman's original study, multiple problems were not used. Similar discrepancies from the standard risk-averse versus risk-seeking reversal pattern were found in other studies (Levin, Gaeth, \& Conlon, 1993; Schneider, 1992). Hence, the gap between the two responses does not always straddle the $50 \%$ line. The traditional pattern of framing effects in Tversky and Kahneman's disease problem may be a special case of a more robust framing effect.

The causal schema introduced into the problems made the two reciprocal sides of the consequence more accessible to the subjects, although the two choices were still represented only in the positive or negative perspective. Is the rationale condition no different from presenting a choice problem in two frames simultaneously as McNeil, Pauker, and Tversky (1988) did? There is certainly some similarity between our manipulation and presenting a problem in both frames simultaneously. But, there are also important differences between providing a causal explanation of why saving 200 lives is equivalent to losing 400 lives and simply stating so. A simple statement of the equivalence rela- tionship placed in the choices to remind the subjects of the reciprocal relationship may or may not be effective in diminishing the framing effect, depending on the specific problems.

Some preliminary pilot studies showed that when the causal mechanism linking the gain to the loss (or vice versa) was evident (such as gaining in one gamble related to loss in another), such a reminder substantially reduced the framing effects. But, when the causal mechanism is not by itself evident (such as in the Tversky and Kahneman's, 1981, disease problem), such a reminder produced very little effect. ${ }^{1}$ Thus, providing an explanatory mechanism is more than making a direct statement about the equivalence relationship. A statement about the equivalence between the gain and the loss which is not supported by an easily understandable account of how and why the two outcomes are equivalent can be characterized as arbitrary. People often have difficulty in understanding arbitrarily defined relations (Cheng \& Holyoak, 1985).

The causal schema introduced may be making the gain and loss relationship come about simultaneously and immediately in a vivid and compelling manner. Whether or not people perceive one event as a part of another or as an unrelated event is very much a matter of subjective perceptual or cognitive structuring (Jou \& Shanteau, 1995). It may be that in order for subjects to perceive the death of 400 people as a consequence of saving 200 people, the 400 deaths have to occur immediately and unambiguously after saving 200 lives. The causal schema in the rationale versions might have provided the contemporaneity and salience in the occurrence of the other event.

The frequency of the reciprocal answers in the norationale condition was lower than in the rationale condition, which indicated that the probability of accessing the other aspect of the information was lower in the former than in the latter. If it can be shown that subjects were less likely to actively use the other side of the information, but were also less likely to provide it when asked, then a stronger case can be made that subjects might not represent the equivalence relation in the no-rationale condition to the same extent as in the rationale condition. The distinction between the two processes is one between accessibility and availability (Tulving \& Pearlstone, 1966). Experiment 2 was designed to determine whether the availability of the other side of the information is also lower in the no-rationale condition than in the rationale condition.

\section{EXPERIMENT 2}

In Experiment 2, subjects were first asked to complete one problem, then do some irrelevant interpolated task, and finally answer a reciprocal question (asking for the amount lost if the subject received a positively framed problem, and vice versa). The purpose of the interpolated task was to attenuate subjects' verbatim memory of the problem, so that the chance that they would immediately compute the reciprocal information post hoc would be reduced. Under the assumption that the chance for a post hoc computation is lessened by the interpolated task, sub- 
jects would be more likely to retrieve the reciprocal information if they had activated that information in processing the problems than if they had not. That is, they should remember better the gist of the information activated or used earlier, after the surface form has been obscured. Since the reciprocal information is assumed to be a coherent part of the schematic story, when the schema is activated, the reciprocal information should also be activated. Hence, we predicted that the chance of providing the correct answer to the reciprocal question would be higher in the rationale than in the no-rationale condition.

\section{Method}

Subjects. One hundred sixty introductory psychology students at the University of Texas-Pan American participated in the experiment for extra credit in the course.

Materials and Design. The materials and design used were the same as in Experiment 1, with the exception of the following: First, each subject completed only one problem (of a certain scenario in one rationale and one frame condition). Second, an unrelated filler questionnaire followed the one-problem questionnaire, to attenuate the subjects' verbatim memory of the problem. Third, a problem asked the subjects a reciprocal question about the amount of life or property saved (for an original negative problem) or amount lost (for an original positive problem). The last sheet of the test booklet contained two questions which asked for the total number of people or properties involved and the number saved or lost that were stated in the original problems. The interpolated task consisted of deciphering the meaning of two rather cryptic, out-of-context passages, one of which, entitled "Doing the Laundry," was adopted from Bransford and Johnson (1972); the other described a car salesman meeting a customer in a showroom. The subjects were asked to write down an interpretation of these passages.

The subjects had three choices for answering the reciprocal question: to give an exact number saved or lost, to give an estimate, or to say they did not know. (Of all correct answers, only 5\% were estimates; they were not considered further.)

There were four independent variables, rationale ( 2 levels), entity ( 2 levels), scenario (4 levels nested within each level of entity), and frame ( 2 levels), for a total of 32 cells. Five subjects were tested in each cell. The rationales for using this completely between-subjects design were, first, to match the design in Tversky and Kahneman's (1981) original study, and second, to determine whether the same pattern of results could be obtained as in Experiment 1, where the entity and scenario variables were within-subjects variables. Since the number of subjects within each cell was only 5 , the four scenarios within each entity were collapsed in data analysis.

Procedure. The general procedure was the same as in Experiment 1 , with the exception of a few variations. Subjects were told that there were four parts to the whole questionnaire, and that they were to complete these parts in the order in which they were presented. They should not return to pages already completed. No time limit was imposed; however, subjects generally spent $8-10 \mathrm{~min}$ on the interpolated task

\section{Results}

Two dependent measures were analyzed, the percentage of the risk-averse choice, and the percentage of the correct reciprocal answers (an answer was scored either as correct or incorrect).

Analysis of the percentage of risk-averse choice. The mean frequencies of the risk-averse choice per scenario for the life and property condition (based on the 5 subjects assigned to each scenario) appear in the top half
Table 2

Mean Frequencies Per Scenario of Risk-Averse Choice and of the Correct Reciprocal Answers by Postexperiment Elicitation in Experiment 2

\begin{tabular}{|c|c|c|c|c|c|c|}
\hline \multirow[b]{2}{*}{ Condition } & \multicolumn{2}{|c|}{ Life } & \multicolumn{2}{|c|}{ Property } & \multicolumn{2}{|c|}{ Combined } \\
\hline & $M$ & $S D$ & $M$ & $S D$ & $M$ & $S D$ \\
\hline
\end{tabular}

Risk-Averse Choice

\begin{tabular}{|c|c|c|c|c|c|c|}
\hline \multicolumn{7}{|l|}{ No-rationale } \\
\hline Positive & 3.3 & 1.0 & 3.8 & 0.5 & 3.5 & \\
\hline Negative & 0.5 & 0.6 & 1.8 & 1.0 & 1.1 & \\
\hline \multicolumn{7}{|l|}{ Rationale } \\
\hline Positive & 1.3 & 1.0 & 3.5 & 0.6 & 2.4 & \\
\hline Negative & 0.8 & 0.5 & 3.3 & 1.5 & 2.0 & \\
\hline \multicolumn{7}{|c|}{ Correct Reciprocal Answers } \\
\hline \multicolumn{6}{|l|}{ No-rationale } & \\
\hline Positive & 2.0 & 0.8 & 2.5 & 1.3 & 2.3 & \\
\hline Negative & 1.8 & 1.0 & 2.0 & 0.8 & 1.9 & \\
\hline \multicolumn{6}{|l|}{ Rationale } & \\
\hline Positive & 4.3 & 1.0 & 4.5 & 1.0 & 4.4 & \\
\hline Negative & 4.8 & 0.5 & 4.3 & 1.0 & 4.5 & \\
\hline
\end{tabular}

Note $\operatorname{Cell} N=5$

of Table 2. As noted earlier, the four scenarios nested within an entity were collapsed to increase the power of the test; therefore, the factor scenario was not used in the analysis. Only significant $(p<.05)$ effects are reported.

The main effect of entity was significant $\left[\chi^{2}(1)=16.99\right.$, $p<.001]$. The choices in the life condition were riskier than in the property condition, which is consistent with the results in Experiment 1; the mean percent of risk-averse choice was 29 for the life-in-danger scenarios, and 62 for the property-in-danger scenarios. The main effect of frame was also significant $\left[\chi^{2}(1)=12.25, p<.001\right]$, with the choice in the positive frame overall more risk averse than in the negative frame. The mean percentage of risk-averse choice was 59 for the positive frame, and 31 for the negative frame.

The crucial rationale $\times$ frame interaction was significant $\left[\chi^{2}(1)=5.66, p<.05\right]$. Within the no-rationale condition, the mean percentage of risk-averse choice was 70 for the positive, and only 22 for the negative frame. These two percentages in the rationale condition were 48 and 40 . Thus, our hypothesis was confirmed that subjects would show significantly greater framing effects in the no-rationale than in the rationale condition. Separate analyses were performed for the no-rationale and rationale conditions. For the no-rationale condition, the framing effect was significant $\left[\chi^{2}(1)=16.29, p<.001\right]$, whereas for the rationale condition, it was not $\left[\chi^{2}(1)=.67, p>.05\right]$. No other effects were significant.

Analysis of the percentages of correct reciprocal answers. The written responses in answering the reciprocal question were counted as correct only if the exact number of lives or amount of property saved or lost was given. The mean frequencies of correct reciprocal answers per scenario given in Experiment 2 are shown in the bottom half of Table 2 . The analysis was performed with the scenarios collapsed. The rationale main effect was significant $\left[\chi^{2}(1)=31.22, p<.001\right]$, with a mean for the no-rationale 
condition of 41 , and for the rationale condition, of 89 . Thus, our hypothesis that the reciprocal side of the information would be more available to the rationale subjects than to the no-rationale subjects was confirmed. No other main effects or interactions reached significance.

The percentage of correct answers to the question about the total amount of lives or properties was 98 for the norationale condition, and 95 for the rationale condition. The percentage of correct answers on the amount saved or lost as stated in the original problem was 96 for the norationale condition, and 98 for the rationale condition. Since the means were so close to being perfect, no analysis was done. Almost all subjects by the end of the whole set of questionnaires still remembered the two original numbers.

\section{Discussion}

The percentages of risk-averse choices from Experiment 2 were consistent with those of Experiment 1, although the framing effect was enhanced a little in the no-rationale condition of Experiment 2 relative to Experiment 1. The smaller framing effect obtained in the no-rationale condition in Experiment 1 may be due to the fact that experience with many problems in Experiment 1 (a within-subjects design) helped the subjects see the implied equivalence relation better. Although the overall percentages of the reciprocal answers elicited in Experiment 2 were higher, the pattern of the percentages across the no-rationale and rationale conditions was the same as in Experiment 1. The fact that the reciprocal information remained more available in the rationale condition after some distracting task than in the no-rationale condition supports the hypothesis that this information was represented or activated to a greater extent in the rationale condition. Thus, high availability of the reciprocal information was coupled with the disappearance of the framing effects, which is consistent with the finding in Experiment 1. Thus, framing effects correlate with low accessibility and availability of the other side of the information.

A formulation of the problem that provided a causal rationale for the mutual entailing of the two aspects of the consequence effectively heightened the accessibility of the other side of the consequence and as a result eliminated the framing effects. In other words, when subjects mentally represented the problems in the structures of Equations 1 and 2 (as shown by the higher rates of spontaneous and elicited reciprocal answers), their responses were no longer inconsistent.

The subjects gave almost perfect answers to the questions posed at the end of the questionnaire about the number of people or amount of property saved or lost as stated in the problems. This fact indicated that the information explicitly given in the problem was still fully available in both rationale conditions when the subjects finished the reciprocal question. Therefore, the large difference in the percentage of the reciprocal answers between the two rationale conditions can be attributed to subjects' failure to make the causal connection between the two aspects of the consequence rather than to the differential levels of availability of the original information.

\section{GENERAL DISCUSSION}

\section{Risk Seeking Toward Life Versus Risk Averse Toward Property}

Subjects showed a risk-averse tendency toward handling property under most of the uncertain conditions, but a risk-seeking attitude toward human life. Such findings suggest that decisions about human life are made differently from economic ones. Perhaps in real life also, judgments about human life follow a principle different than do economic decisions. In the no-rationale life-in-danger condition, the typical framing effect pattern prevailedthat is, risk aversion in the positive but risk seeking in the negative frame. In the rationale condition, however, decisions about human lives consisted of risk seeking in both frames.

We obtained significant framing effects for both lifeand property-in-danger scenarios in the no-rationale condition, which was consistent with the findings in the literature. What was not consistent with the typical findings was a predominantly risk-seeking tendency toward human life but a risk-averse tendency toward property in the rationale condition, regardless of the frames. In the following section, we will elaborate on the explanation given earlier to account for the different tendencies toward human life and property in the rationale condition.

In the rationale condition, subjects causally linked the positive to the negative consequence and vice versa. Hence, they were making judgments on a single holistic representation of the problem. However, they followed different principles concerning life and property. The principle for life may be called the noninterchangeability principle; that is, no life should be sacrificed for the sake of another life, and so every individual should have an equal chance of surviving or being saved (this favors the risky choice). The principle for property may be called the interchangeability principle; that is, it is all right or even desirable to save a portion of the property with certainty, at the expense of other portions. Since the mental representation of the two versions of a problem was identical in the rationale condition, the response patterns across the two frames became consistent - hence the disappearance of the framing effects. However, the two principles dictate reverse tendencies-hence the different response patterns for life and property scenarios.

An interesting question would be why the two principles did not operate, or not operate in the same way, in the norationale condition. We suggest that, in the no-rationale condition, the causal relationship between the two aspects of the consequence was not recognized, and as a result, subjects were not representing the two versions of the problems in an identical form. Since it did not occur to the majority of subjects that saving 200 lives meant causing 400 deaths (i.e., no interchange occurred), the noninterchangeable principle was not applied. Hence there is noth- 
ing wrong with saving 200 lives for sure. By the same token, the majority of subjects in the no-rationale condition was not aware that losing $\$ 400$ meant gaining \$200. Therefore, no exchange of loss for gain took place, and the interchangeability principle was inapplicable.

In sum, the activation of the schema gave rise to the realization of the equivalence relation, which in turn led to the application of the different interchangeability principles for different tasks. The lack of a causal link between gain and loss in the no-rationale condition makes the interchangeability factor irrelevant. Under those circumstances, people may be following a same decision principle for both life and property, which they thought would maximize gain and minimize loss.

\section{Contextual Constraints and Ethical Considerations}

The additional contextual information introduced into the problems might also have introduced some extraneous factors into the judgments and complicated the interpretation of the results. For example, making decisions in the rationale versions might have incurred a greater sense of responsibility than did making decisions in the no-rationale versions. Could there have been any contribution from factors of responsibility and ethics, even when we told the subjects the decision was anonymous? The answer is probably yes. Does such a possibility render the findings worthless? The answer is no. All decisions involve responsibility and ethics, especially when human life and property are at stake. No decision is completely responsibility free, including the ones in Tversky and Kahneman's (1981) original study; only more or less responsibility is involved. In real life, decisions completely free of responsibility and ethical implications are more of an exception than the norm. External validity has been a long-standing concern about decision-making studies conducted in the laboratory (Corbin, 1980; Ebbesen \& Konečni, 1980; Levin et al., 1988; Winkler \& Murphy, 1973). There is more to the psychology of decision making than simply judging statistical numbers anonymously in an abstract format. The induction of the causal schema brought relevant life experience to bear on the judgment and hence raised the ethical consciousness level of the subjects. ${ }^{2}$

Subjects obviously used different ethical standards in dealing with human life and property. The effect attributable to the use of different ethical standards was reflected in the overall riskiness levels of the decisions. However, this effect was unrelated to the effects of framing. The framing effects were most sensitive to the rationale variable. The effects of scenarios reflected the idiosyncrasies of the individual problems. However, beyond the effects of ethics and idiosyncrasies, there was a systematic change in the pattern of the data from the no-rationale to the rationale versions - the diminishing of the framing effects.

\section{An Information-Processing View of Framing Effects}

Tversky and Kahneman (1981) based their explanation of framing in decision making on some psychophysical functions relating psychological values to objective values of entities. Their theory gives an account of why people have a risk-averse tendency in the gain context and a riskseeking tendency in the loss context. In the present study, we are not concerned with why people have each tendency under each frame. Instead, we are concerned with the cognitive reason why the two perspectives do not connect with each other. In everyday life, it is commonplace that a choice produces opposite effects. For example, buying a high-quality but expensive car will result in owning a trouble-free and comfortable vehicle but at the same time in paying a higher price. Buying a cheap car will have the reverse effects. Living in a big city will give one better access to cultural and other facilities but also the disadvantages of air pollution and high crime rates. From the informationprocessing point of view, framing is a form of manipulating the salience or accessibility of different aspects of information. One aspect of a consequence can be highlighted and the other deemphasized, or even equivocated. A decision is the product of the combined impressions about various aspects of information which are often unequally weighted (Birnbaum \& Jou, 1990). The function of introducing the rationale is to ensure that the underweighted side of the information is given greater weight.

An important process in comprehension of text is pragmatic inference (Graesser \& Bower, 1990; Graesser, Singer, \& Trabasso, 1994; Harris, 1981). Some information is implied rather than explicitly stated, which the comprehender will fill in through inference. The mental access to the other side of the outcome in processing a frame problem is analogous to an inference process, whereby information not explicitly stated is inferred on the basis of some general knowledge (schemas) that comprehenders possess about the events in question. The no-rationale version of the problems may be formulated so that inferring the other side of the consequence is less likely to occur, perhaps because of a failure to connect to the general knowledge base. The rationale version may have provided a link to that missing knowledge base. The higher frequencies of actively using the "inferred" information in the decision process and the higher level of availability after the decision has been made suggest that the other aspect of the information has been accessed or activated in the rationale condition.

If every event in the world has an objective state (which is questionable) that can be represented from a positive and a negative view, prospect theory predicts how people would behave within each frame. The information-processing view we are suggesting is concerned with the cognitive processes involved, with the nature of the limiting effects of framing, and with why subjects in Tversky and Kahneman's (1981) original study behaved inconsistently.

\section{Performance Limitation and Problem Representation}

Problems posed to the subjects in some studies which demonstrate inconsistency in decision making involve lengthy and complicated computations of aggregating products of payoffs and their associated probabilities 
(Dawes, 1988; Hogarth, 1987; Kahneman \& Tversky, 1986; Redelmeier \& Tversky, 1992; Tversky \& Kahneman, 1981). Many of the computations may well be beyond the capacity of ordinary people's mental calculation. People may selectively attend to some salient aspects of such complex numerical information and overlook others because a complete computation of the outcomes exceeds their attentional and short-term memory span. Thus, some judgmental inconsistencies under those circumstances may derive from performance limitations rather than from some fundamental deficiencies in the competence of the human cognitive system (Cohen, 1981; Einhorn \& Hogarth, 1981; Hogarth, 1981, 1987; Jungermann, 1986). It is always possible to set up an experimental condition which capitalizes on memory and attention limitations. The inconsistent judgments and decisions demonstrated in these studies, therefore, are not necessarily indications of fundamental human irrationality.

Another type of experimental question used to demonstrate inconsistency in choice behaviors is represented by the disease problem examined in the present study. The lostticket problem of Kahneman and Tversky (1984) ("Would you buy another ticket if you lose the original ticket which cost \$10?" versus "if you lose $\$ 10$ ?") can also be considered to be similar. In this type of question, no complicated mental calculations are required. The apparent inconsistencies in decisions across the two frames may have derived from the fact that subjects and experimenters have different representations or understandings of the problem (Berkeley \& Humphreys, 1982; Jungermann, 1986). Too much emphasis has been put on the errors that the subjects make, and too little on subjects' internal representations of the problems (Berkeley \& Humphreys, 1982; Jungermann, 1986; Wagenaar, Keren, \& Lichtenstein, 1988). In the present paper, we have presented an effort to address the question of problem representation and its effect on framing effects.

In some studies, the inconsistency or irrationality conclusion has been based on the assumption that the equivalence relationship holds between the two outcomes of a decision. In our study, we considered whether there was enough constraining information in the scenarios to justify such an assumption. Subjects certainly can perform the simple mental arithmetic involved in Equations 1 and 2. If so, why did they make the choices they did? Probably because they did not think of, or at least did not interpret the problem in light of, the equivalence relation. The fact that subjects in the no-rationale condition accessed the other side of the consequence less frequently, and that they could not answer the reciprocal question as well as the subjects in the rationale condition, is evidence that they did not construe the problem in the way intended by the experimenters. For subjects to perceive this relationship, it needs to be embedded in a schematic framework.

\section{Ecological Considerations}

The results from the rationale condition suggest that framing effects in real life may not be as prevalent as some decision studies have led us to believe. In real life, there may be many contextual cues and constraints (including responsibilities and ethics) which make it easier for decision makers to see the relationship between different aspects of the consequence of a decision (Hogarth, 1981, 1987). As a result, the decision is less likely to be based on the superficial form in which the problem is posed. Our conclusion is in agreement with other reasoning and judgment studies in which it has been concluded that people are more likely to make judgments and decisions in accord with the normative rules when a problem is presented in a familiar and realistic context (Agnoli \& Krantz, 1989; Cox \& Griggs, 1982; Gigerenzer, Hell, \& Blank, 1988; JohnsonLaird, Legrenzi, \& Legrenzi, 1972; Wolford, Taylor, \& Beck, 1990).

One explanation for the framing effect is that people take different reference points when a problem is represented in different frames (Dawes, 1988; Helson, 1948; Tversky \& Kahneman, 1981). The reversal of preference can also be compared to a perceptual figure-ground reversal (Einhorn \& Hogarth, 1981). In both cases, the reversal is possible only if there is enough ambiguity, perceptual or cognitive, in the stimulus to allow two percepts to be created. In many subjects' minds, the final state of the 400 people who are supposed to die as a result of saving 200 people may be simply unknown or undetermined. We have shown that framing effects can be eliminated or greatly reduced when the level of ambiguity is reduced by the introduction of a causal schema.

In real life, choices are usually delayed until uncertainty is resolved or reduced below a certain threshold (Hogarth, 1981). In real-life situations, according to Hogarth (1981), inconsistencies caused by adopting different reference points in decision making under uncertainty are not irrational. In fact, he argued that accepting the status quo as a reference point for choices was ecologically functional when there was uncertainty in the situation-and this was exactly the case in the no-rationale versions of the problems. Since subjects were unable to determine the fate of the other portion of the people or property (i.e., unable to answer the reciprocal questions), their responses might actually have reflected an adaptive strategy that could be used in real life in an uncertain situation.

Also, to frame choices in terms of total or final consequences in real life can be counterproductive. For instance, a tendency to frame a past state as the reference point can lead to such counteradaptive behaviors as commitments to sink costs, since abandoning an unfinished project would be perceived as the acceptance of a sure loss (Arkes \& Blumer, 1985; Dawes, 1988; Shanteau \& Harrison, 1991). Another reason why using the status quo as a reference point might be adaptive is that it may be difficult in many circumstances to define an objective state of affairs. For instance, there is often no standard way of setting a starting point (or setting an aspiration level, for that matter) for the assessment of total outcome. One can always regress to an earlier point in counting the total assets. However, an arbitrary or subjective point has to be chosen somewhere along the line as the starting point for assessing an outcome of a decision (Jou \& Shanteau, 1995). The current 
state of affairs seems to be a reasonable choice for that arbitrary starting point.

If the ambiguity or uncertainty about the relationship between the gains and the reciprocal losses in Tversky and Kahneman's original problem is representative of some real-life situations, and if the subjects are forced to make a choice under such uncertainty, then adopting the status quo as the reference point is perhaps reasonable. Such a choice implies that the decision maker is realistically minded rather than rigidly consistent. Such rigidity is, in fact, a sign of maladaptiveness (Korchin, 1976; Shakow, 1977). Furthermore, we demonstrated that subjects were influenced little by the manipulation of the frame when there was a more definitive description of both the desirable and the undesirable aspects of a decision. Just as sensory adaptation is ecologically functional, cognitive adaptation to a status quo may be an ecologically functional strategy in an ever-changing and uncertain environment. But, as we have shown, people adopt this strategy only when there is substantial uncertainty in the situation.

\section{REFERENCES}

Agnoli, F., \& Krantz, D. H. (1989). Suppressing natural heuristics by formal instruction: The case of the conjunction fallacy. Cognitive Psychology, 21, 515-550.

ARkes, H. R., \& Blumer, C. (1985). The psychology of sunk cost. Organizational Behavior \& Human Decision Processes, 35, 124-140.

BERKELEY, D., \& HuMPHREYS, P. (1982). Structuring decision problems and the "bias heuristic." Acta Psychologica, 50, 201-252.

Birnbaum, M. H., \& Jou, J. (1990). A theory of comparative response times and "difference" judgments. Cognitive Psychology, 22, 184-210

Bradburn, N. M. (1982). Question-wording effects in surveys. In R. M Hogarth (Ed.), Question framing and response consistency (pp. 51-63). San Francisco: Jossey-Bass.

Bransford, J. D., \& JOHNSON, M. K. (1972). Contextua! prerequisites for understanding: Some investigations of comprehension and recall. Joumal of Verbal Learning \& Verbal Behavior, 11, 717-721.

Cheng, P. W., \& Holyoak, K. J. (1985). Pragmatic reasoning schemas. Cognitive Psychology, 17, 391-416.

COHEN, L. J. ( 1981). Can human irrationality be experimentally demonstrated? Behavioral \& Brain Sciences, 4, 317-370.

Corbin, R. M. (1980). Decisions that might not get made. In T. S. Wallsten (Ed.), Cognitive processes in choice and decision behavior (pp. 47-67). Hillsdale, NJ: Erlbaum.

Cox, J. R., \& GrigGs, R. A. (1982). The effects of experience on performance in Wason's selection task. Memory \& Cognition, 10, 496-502.

DAWES, R. M. (1988). Rational choice in an uncertain world. New York: Harcourt Brace Jovanovich.

EBBEsen, E. B., \& KONEČNI, V. J. (1980). On the external validity of decision-making research: What do we know about decisions in the real world? In T. S. Wallsten (Ed.). Cognitive processes in choice and decision hehavior (pp. 21-45). Hillsdale, NJ: Erlbaum.

EINHORN, H. J., \& HogarTh, R. M. (1981). Behavioral decision theory: Processes of judgment and choice. Annual Review of Psychology, 32, $53-88$

Gigerenzer, G., Hell, W., \& Blank, H. (1988). Presentation and content: The use of base rates as a continuous variable. Journal of Experimental Psychology: Human Perception \& Performance, 14, 513-525.

Goldstein, W. M., \& EINHORN, H. J. (1987). Expression theory and the preference reversal phenomena. Psychological Review, 94, 236-254.

Graesser, A. C., \& Bower, G. H. (EDS.) (1990). Inferences and text comprehension. San Diego, CA: Academic Press.

Graesser, A. C., \& Nakamura, G. V. (1982). The impact of a schema on comprehension and memory. In G. H. Bower (Ed.), The psychology of learning and motivation: Advances in research and theory (Vol. 16, pp. 59-109). New York: Academic Press.

Graesser, A. C., Singer, M., \& Trabasso, T. (1994). Constructing inferences during narrative text comprehension. Psychological Review, 101, 371-395.

Greenwald, A. G., Pratkanis, A. R., Leippe, M. R., \& BaumgardNER, M. H. (1986). Under what conditions does theory obstruct research progress? Psychological Review, 93, 216-229.

HARRIS, R. J. (1981). Inferences in information processing. In G. H, Bower (Ed.), The psychology of learning and motivation: Advances in reseach and theory (Vol. 15, pp. 81-128). New York: Academic Press.

HeLsON, H. (1948). Adaptation-level as a basis for a quantitative theory of frames of reference. Psychological Review, 55, 297-313.

Hershey, J. C., \& SChoemaker, P. J. H. (1980). Risk taking and problem context in the domain of losses: An expected utility analysis. Journal of Risk \& Insurance, 47, 111-132.

Hogarth, R. M. (1981). Beyond discrete biases: Functional and dysfunctional aspects of judgmental heuristics. Psychological Bulletin, 90, 197-217

HogarTH, R. M. (1987). Judgment and choice: The psychology of decision (2nd ed.). New York: Wiley.

Johnson-LaIRD, P. N., LegrenZI, P., \& Legrenzi, M. (1972). Reasoning and a sense of reality. British Journal of Psychology, 63, 395-400.

Jou, J., \& Shanteau, J. (1995). The gestalt and dynamic processes in decision making. Behavioural Processes, 33, 305-318.

JungermanN, H. (1986). The two camps on rationality. In H. R. Arkes \& K. R. Hammond (Eds.), Judgment and decision making: An interdisciplinary reader (pp. 627-641). New York: Cambridge University Press.

Kahneman, D., \& Tversky, A. (1979). Prospect theory: An analysis of decision under risk. Econometrica, 47, 263-291.

Kahneman, D., \& TVERSKY, A. (1984). Choices, values, and frames. American Psychologist, 39, 341-350.

KahNeman, D., \& TVERSKY, A. (1986). Choice, values, and frames. In H. R. Arkes \& K. R. Hammond (Eds.), Judgment and decision making: An interdisciplinary reader (pp. 195-210). New York: Cambridge University Press.

KoRCHIN, S. J. (1976), Modern clinical psychology. New York: Basic Books.

Levin, 1. P., Gaeth, G. J., \& Conlon, A. B. (1993, November). A review and synthesis of inconsistent findings in framing effects research. Paper presented at the meeting of the Psychonomic Society, Washington, DC.

LeVin, I. P., SChNitTIER, S. K., \& Thee, S. L. (1988). Information framing effects in social and personal decisions. Journal of Experimental Social Psychology, 24, 520-529.

Lichrenstein, S., \& Slovic, P. (1973). Response-induced reversals of preference in gambling: An extended replication in Las Vegas. Journal of Experimental Psychology, 101, 16-20.

MCNEIL, B. J., PAUKER, S. G., \& TVERSKY, A. (1988). On the framing of medical decisions. In D. E. Beli, H. Raiffa, \& A. Tversky (Eds.), Decision making: Descriptive, normative, and prescriptive interactions (pp. 562-568). New York: Cambridge University Press.

ReDELMEIER, D. A., \& TVERSKY, A. (1992). On the framing of multiple prospects. Psychological Science, 3, 191-193.

SAS INSTITUTE (1985). SAS user's guide: Statistics (Version 5 edition). Cary, NC: Author

SChANK, R. C., \& ABELSON, R. (1977). Scripts, plans, goals, and understanding. Hillsdale, NJ: Erlbaum.

SCHNEIDER, S. L. (1992). Framing and conflict: Aspiration level contingency, the status quo, and current theories of risky choice. Journal of Experimental Psychology: Learning, Memory, \& Cognition, 18, $1040-$ 1057

SCHNEIDER, S. L., \& LOPES, L. L. (1986). Reflection in preferences under risk: Who and when may suggest why. Journal of Experimental Psychology: Human Perception \& Performance, 12, 535-548.

SHaKow, D. (1977). Segmental set: The adaptive process in schizophrenia. American Psychologist, 32, 129-139.

SHANTEAU, J., \& HaRRISON, P. (1991). The perceived strength of an im- 
plied contract: Can it withstand financial temptation? Organizational Behavior \& Human Decision Processes, 49, 1-21

Slovic, P., Fischioff, B., \& Lichtenstein, S. (1982). Response mode, framing, and information-processing effects in risk assessment. In R. M. Hogarth (Ed.), Question framing and response consistency (pp. 21-36). San Francisco: Jossey-Bass.

Tulving, E., \& PeArlstone, Z. (1966). Availability versus accessibility of information in memory for words. Journal of Verbal Learning \& Verbal Behavior, 5, 381-391.

TVERSKY, A., \& KaHNEMAN, D. (1973). Availability: A heuristic for judging frequency and probability. Cognitive Psychology, 5, 207-232.

TVersky, A., \& Kahneman, D. (1981, January 30). The framing of decisions and the psychology of choice. Science, 211, 453-458.

WagenaAr, W. A., Keren, G., \& Lichtenstein, S. (1988). Islanders and hostages: Deep and surface structures of decision problems. Acta Psychologica, 67, 175-189.

Wason, P. C. (1966). Reasoning. In B. M. Foss (Ed.), New horizons in psychology (Vol. 1, pp. 135-151). Harmondsworth, U.K.: Penguin. WASON, P. C., \& JOHNSON-LAIRD, P. C. (1972). Psychology of reasoning. Cambridge, MA: Harvard University Press.

WinKLER, R. L., \& MURPhy, A. H. (1973). Experiments in the laboratory and the real world. Organizational Behavior \& Human Performance, 10, 252-270.

WOLFORD, G., TAYLOR, H. A., \& BECK, J. R. (1990). The conjunction fallacy? Memory \& Cognition, 18, 47-53.

\section{NOTES}

1. In one of our pilot studies, subjects were told to imagine that they had already lost $\$ 600$ in a first gamble. A second gamble was presented to them in either a gain or a lose frame. Substantial framing effects were obtained. In a follow-up experiment, an explicit reminder was provided about the $\$ 600$ already lost in the first gamble, and subjects were told that gaining $\$ 200$ in the second gamble was therefore equivalent to losing $\$ 400$ and vice versa from the viewpoint of total assets. Framing effects were effectively eliminated. The risk-averse choice was preferred by the majority in both frames, which is consistent with our other data on property problems. In another initial experiment, an explicit reminder was given in the disease problem, but only a weak effect was obtained. The details of the studies are available upon request.

2. In one of our pilot studies, we instructed the subjects to imagine that they were the captain of a sinking ship with either human lives or precious metal in danger. In another experiment, the same scenarios were used, except that subjects were not instructed to imagine that they were the captains. No difference in subjects' responses was found. Thus, personal responsibility did not seem to play a significant role in this kind of hypothetical decision making. The details of the studies are available upon request.

\section{APPENDIX}

\section{No-Rationale Condition}

\section{Life in Danger}

Disease. Imagine that the U.S. is preparing for an outbreak of an unusual disease, which is expected to kill 600 people. Two alternative programs to combat the disease have been proposed.

\section{Positive Frame}

Option A. If Program A is adopted, 200 people will be saved. Option B. If Program B is adopted, there is a one-third probability that all 600 people will be saved, and a two-thirds probability that no people will be saved.

\section{Negative Frame}

Option A. If Program A is adopted, 400 people will die.

Option B. If Program B is adopted, there is a one-third probability that nobody will die, and a two-thirds probability that all 600 people will die.
Shipwreck. Imagine that a ship hits a water mine in the middle of the ocean. Six hundred passengers on board are in danger. Two options are proposed.

\section{Positive Frame}

Option A. If Option A is adopted, 200 passengers will be saved. Option B. If Option B is adopted, there is a one-third probability that all 600 passengers will be saved, and a two-thirds probability that no passenger will be saved.

\section{Negative Frame}

Option A. If Option A is adopted, 400 passengers will die. Option B. If Option B is adopted, there is a one-third probability that no passenger will die, and a two-thirds probability that all 600 passengers will die.

Volcano. Imagine that a volcano is erupting on a volcanic island in the Pacific Ocean. Six hundred residents of the island are in danger. Two alternatives are proposed.

\section{Positive Frame}

Option A. If Option A is adopted, 200 islanders will be saved.

Option B. If Option B is adopted, there is a one-third probability that all 600 islanders will be saved, and a two-thirds probability that no islander will be saved.

\section{Negative Frame}

Option $A$. If Option A is adopted, 400 islanders will die.

Option B. If option B is adopted, there is a one-third probability that no islander will die, and a two-thirds probability that all 600 islanders will die.

Hurricane. Imagine that somewhere in the Caribbean Sea a group of small islands is located in an area often ravaged by hurricanes. Each year, many lives are lost because of the hurricanes. An estimated 300 people's lives will be in danger in the next hurricane season. Two alternatives are proposed.

\section{Positive Frame}

Option A. If Alternative A is adopted, an estimated 100 lives will be saved in the next hurricane season.

Option B. If Alternative B is adopted, there is a one-third probability that all 300 people's lives will be saved, and a two-thirds probability that none of the 300 lives will be saved.

\section{Negative Frame}

Option $A$. If Alternative A is adopted, an estimated 200 lives will be lost in the next hurricane season.

Option B. If Alternative B is adopted, there is a one-third probability that none of the 300 people's lives will be lost, and a twothirds probability that all the 300 lives will be lost.

\section{Properties in Danger}

Shipwreck. Imagine that a cargo ship hits a water mine in the middle of the ocean. Six hundred boxes of a precious metal on board are jeopardized. Two options are proposed

\section{Positive Frame}

Option A. If Option A is adopted, 200 boxes of the precious metal will be saved.

Option B. If Option B is adopted, there is a one-third probability that all 600 boxes of the precious metal will be saved, and a two-thirds probability that none of the precious metal will be saved.

\section{Negative Frame}

Option A. If Option A is adopted, 400 boxes of the precious metal will be lost. 
Option B. If Option B is adopted, there is a one-third probability that no precious metal will be lost, and a two-thirds probability that all 600 boxes of the precious metal will be lost.

Apartment. Imagine that Mary cannot fulfill a contract she signed with an apartment manager. Her deposit of $\$ 600$ is in jeopardy. There are two alternatives.

\section{Positive Frame}

Option A. If she chooses Option A, she will get $\$ 200$ back.

Option B. If she chooses Option B, there is a one-third probability that she will get all $\$ 600$ back, and a two-thirds probability that she will get nothing back.

Which option would you favor (that is, in the best interest of Mary)?

\section{Negative Frame}

Option A. If she chooses Option A, she will lose $\$ 400$.

Option B. If she chooses Option B, there is a one-third probability that she will lose nothing, and a two-thirds probability that she will lose all $\$ 600$ of deposit.

Which option would you favor (that is, in the best interest of Mary)?

Investment. Imagine that John invested $\$ 60,000$ in a company. The company's financial situation is in jeopardy, and so is John's investment. Two alternatives are available.

\section{Positive Frame}

Option A. If Option A is adopted, John will get back $\$ 20,000$.

Option B. If Option B is adopted, there is a one-third probability that John will get all $\$ 60,000$ of the investment back, and a two-thirds probability that he will get nothing back.

\section{Negative Frame}

Option A. If Option A is adopted, John will lose $\$ 40,000$.

Option B. If Option B is adopted, there is a one-third probability that John will lose nothing, and a two-thirds probability that John will lose all $\$ 60,000$ of the investment.

Which option would you favor (that is, in John's best interest)?

Selling Assets. Imagine that a chemical company has recently been forced to go out of business. The total assets of the company are worth $\$ 240,000$. Two alternatives for selling the properties are proposed.

\section{Positive Frame}

Option A. If Alternative $\mathrm{A}$ is adopted, the owner will recover $\$ 80,000$.

Option B. If Alternative B is adopted, according to estimates, there is a one-third probability that all $\$ 240,000$ will be recovered. and a two-thirds probability that zero assets will be recovered.

Negative Frame

Option A. If Alternative A is adopted, the owner will lose $\$ 160,000$.

Option B. If Alternative B is adopted, according to estimates, there is a one-third probability that zero assets will be lost, and a two-thirds probability that all $\$ 240,000$ of the assets will be lost.

\section{Rationale Condition}

\section{Life in Danger}

Disease. Imagine that the U.S. has been attacked by an unusual and deadly disease. Without treatment, a person who has contracted the disease will die in a few days. Six hundred people have been diagnosed as having contracted the disease. Some substance, extracted from living human organs and extremely difficult to obtain, can cure the disease. Unfortunately, there is only enough of this substance for 200 people. No additional source of this substance will become available for at least 18 months, and no other cure or treatment will be found in at least the next two decades. If the patient receives an insufficient dose, there is a chance that the patient may live or may die. Two alternatives are proposed.

\section{Positive Frame}

Option $A$. The total amount of substance is applied to 200 of the patients. If this is done, 200 people will be saved.

Option B. The total amount of substance is shared among the 600 patients. If this is done, there is a one-third probability that all 600 patients will be saved, and a two-thirds probability that nobody will be saved.

\section{Negative Frame}

Option A. Four hundred patients will not receive the substance, so that 400 people will die.

Option B. The total substance is shared among the $600 \mathrm{pa}-$ tients, so that there is a one-third probability that nobody will die, and a two-thirds probability that all 600 patients will die.

Shipwreck. Imagine that a ship hits a water mine, and is quickly sinking in the middle of the ocean. There are 600 passengers on the ship, but the lifeboat's maximum capacity is only 200 people. It is known that when the lifeboat is overloaded, there is a chance that the boat will capsize. The ocean water is at the freezing temperature. Anybody falling into the water will surely be frozen to death within half an hour. There is no chance that any rescue will come, since the radio is completely damaged and the night is pitch dark. Two alternatives are proposed.

\section{Positive Frame}

Option A. Two hundred passengers go on board the life boat so that 200 people will be saved.

Option B. All 600 passengers go on board the lifeboat, so that there is a one-third probability of saving all of them, and a twothirds probability of saving none of them should the lifeboat capsize.

\section{Negative Frame}

Option A. Four hundred of the passengers will not go on board the lifeboat, so that 400 people will die.

Option B. All 600 passengers go on board the lifeboat, so that there is a one-third probability that nobody will die, and a twothirds probability that all 600 passengers will die should the lifeboat capsize.

Volcano. Imagine that the volcano on a volcanic island somewhere in the middle of the Pacific Ocean is erupting. It is predicted that a catastrophic explosion can occur soon, which will completely annihilate the whole island along with everything on it. There are 600 residents scattered over the island. Residents near the coast are told the danger and are boarding an evacuation ship. But with no modern communication facilities on the island, the inland residents have not been informed of the imminent disaster and are not moving. Two alternatives are proposed.

\section{Positive Frame}

Option $A$. The ship leaves immediately, so that 200 people will be saved.

Option $B$. The ship waits until the inland residents are all reached, and brought to the ship. If this option is to be carried 
out, there is a one-third probability that all 600 islanders will be saved, and a two-thirds probability that nobody will be saved should the volcano burst into a full explosion.

\section{Negative Frame}

Option $A$. The ship will not wait for the 400 inland residents, so that 400 people will die.

Option $B$. The ship waits until the inland residents are all reached, and brought to the ship. If this is done, there is a onethird probability that nobody will die, and a two-thirds probability that all islanders will die should the volcano burst into a full explosion.

Hurricane. Imagine that somewhere in the Caribbean Sea a group of small islands is located in an area often ravaged by hurricanes. Each year, many lives are lost because of the hurricanes. Common causes of death were the collapse of buildings and houses, and the flood. Old buildings and houses and lack of manpower for the rescue teams are the main problems. Rebuilding these buildings and houses would be a safer measure than just repairing or reinforcing them, but it costs much more. The government has only limited funds to deal with these problems. The question is whether to spend all the limited money on one island or to distribute it among all islands. An estimated 300 people's lives will be in danger in the next hurricane season. Two solutions are proposed.

\section{Positive Frame}

Option A. Use the limited funds to rebuild the shaky buildings and houses, and double the rescue force on one of the islands, so that an estimated 100 lives on this island will be saved in the next hurricane season.

Option B. Distribute the limited funds to all islands to repair the old buildings and houses, and to make a slight increase in the rescue force on all islands, so that there is a one-third probability that all 300 people's lives will be saved if the hurricanes are not too strong, and a two-thirds probability that none of the 300 lives will be saved if the hurricanes turn out to be very strong.

\section{Negative Frame}

Option A. None of the islands except one will receive any funds to repair the buildings and to increase the rescue force, so that an estimated 200 lives will be lost in the next hurricane season.

Option B. Distribute the limited funds to all islands to repair the old buildings and houses, and to make a slight increase in the rescue force on all islands, so that there is a one-third probability that none of the 300 lives will be lost if the hurricanes are not too destructive, and a two-thirds probability that all 300 lives will be lost if the hurricanes turn out to be very strong.

\section{Property in Danger}

Shipwreck. Imagine that a cargo ship hits a water mine and is quickly sinking in the middle of the ocean. There are 600 boxes of a precious metal on the ship. There is only one boat with a maximum capacity load of 200 boxes of the metal. It is known that when the boat is overloaded, there is a chance that the boat will capsize. There is no chance that any rescue will come since the radio is completely damaged and the night is pitch dark. Two alternatives are proposed.

\section{Positive Frame}

Option $A$. Two hundred boxes of the precious metal will be put on the boat so that 200 boxes of the precious metal will be saved.

Option B. All 600 boxes of the precious metal will be put on the boat, so that there is a one-third probability of saving all the boxes of the precious metal, and a two-thirds probability of saving none of them should the boat capsize.

\section{Negative Frame}

Option A. Four hundred boxes of the precious metal will not be put on the boat, so that 400 boxes of the precious metal will be lost.

Option B. All 600 boxes of the precious metal will be put on the boat, so that there is a one-third probability of losing none of the boxes of the precious metal, and a two-thirds probability of losing all of them should the boat capsize.

Apartment. Imagine that Mary has signed a 1-year contract for an apartment in town, and paid a deposit of $\$ 600$ to the manager of the apartment. Something unexpected came up which made it necessary for her to live on campus, and therefore impossible to fulfill her contract. According to the terms of the contract, she can choose one of two options should she not be able to fulfill the contract: she can simply cancel the contract and get a portion of the deposit back, or hopefully get all the deposit back by trying to find someone to take her apartment by putting a free ad in the campus newspaper, but, there is no guarantee that such a replacement will be found. (Note that according to the contract, a tenant may not try one option and then switch to the other, or put the ad anywhere else.)

\section{Positive Frame}

Option $A$. Just cancel the contract with the manager. If she chooses this option, she will get $\$ 200$ back.

Option B. Put an ad in the campus newspaper. According to past statistics, she has a one-third probability of finding someone to take her place within the period allowed by the manager, in which case, she will get back all the $\$ 600$ of deposit, and a two-thirds probability of finding nobody to take her apartment, in which case, she will get back nothing of the $\$ 600$ deposit.

Which option would you favor (that is, in Mary's best interest)?

\section{Negative Frame}

Option $A$. Not go to find someone to take over her apartment. If she chooses this option, she will lose $\$ 400$.

Option B. Put an ad in the campus newspaper. According to past statistics, she has a one-third probability of finding someone to take her place within the period allowed by the manager, in which case, she will lose none of the $\$ 600$ of deposit, and a twothirds probability of finding nobody to take her apartment, in which case, she will lose all $\$ 600$ of deposit.

Which option would you favor (that is, in Mary's best interest)?

Investment. Imagine that John invested $\$ 60,000$ in a company. The company is having financial problems. Two options are being considered: either the company declares bankruptcy right now, in which case it will pay back a portion of the $\$ 60,000$ to John, or it continues to hold out, in which case, the condition can make a turn either for better or for worse.

\section{Positive Frame}

Option $A$. The company declares bankruptcy, and John gets back $\$ 20,000$ of his total investment.

Option B. The company holds out. If this option is taken, there is a one-third probability that John will get back all $\$ 60,000$ of his investment, and a two-thirds probability that he will get nothing back should the company's financial situation get worse.

Which option would you favor (that is, in John's best interest)?

\section{Negative Frame}

Option A. The company does not continue to hold out, and John loses $\$ 40,000$ of his investment. 
Option B. The company holds out. If this option is taken, there is a one-third probability that John will lose nothing, and a twothirds probability that John will lose all $\$ 60,000$ of his investment should the company's financial situation get worse.

Which option would you favor (that is, in John's best interest)?

Selling assets. Imagine that a chemical company has been recently forced to go out of business. The total assets of the company are worth $\$ 240,000$. Right now, there is a buyer who is willing to buy the total assets at one third of the value, i.e., for $\$ 80,000$. Assume that there are two proposals for selling the company: either sell it to this buyer, or wait for a potential buyer who would be willing to pay $\$ 240,000$.

\section{Positive Frame}

Option A. Just sell it to this present buyer, so that the owner will recover $\$ 80,000$.
Option $B$. Wait for a buyer willing to pay the total price. According to estimates, there is a one-third probability that such a buyer will appear, so all $\$ 240,000$ will be recovered, and a twothirds probability that no such a buyer will appear, so zero assets will be recovered.

\section{Negative Frame}

Option A. Not wait for a buyer willing to pay the total price, so the owner will lose $\$ 160,000$.

Option $B$. Wait for a buyer willing to pay the total price. According to estimates, there is a one-third probability that such a buyer will appear, so zero assets will be lost, and a two-thirds probability that no buyer will appear, so all $\$ 240,000$ of the assets will be lost.

(Manuscript received September 14, 1994; revision accepted for publication January 5,1995 .) 